\title{
Duodenal Extraskeletal Osteosarcoma
}

National Cancer Institute

\section{Source}

National Cancer Institute. Duodenal Extraskeletal Osteosarcoma. NCI Thesaurus. Code C82972.

An extraskeletal osteosarcoma affecting the duodenum. 